\title{
Effect of interlaminar flaw on composite panel behaviour under acoustic loading
}

\author{
Jiři Běhal ${ }^{1,1}$, Pavel Zděnek ${ }^{1}$ \\ ${ }^{1}$ VZLU - Czech Aerospace Research Centre, Strength of Structures Department, Beranových 130, \\ 19905 Prague 9, Czech Republic
}

\begin{abstract}
A design of airframe structures has to be accompanied by material properties testing. Special focus has to be laid on high frequency fatigue behaviour. The acoustic fatigue test equipment for grazing wave's incidence was designed based on the FE analyses. Flat composite panels were designed and manufactured using the Hexply 8552/AGP193-PW prepreg with different fiber lay-ups and with simulation of production imperfections or operational damage. Dynamic behaviour of panels was tested using three regimes of acoustic loading: white noise spectrum, engine noise spectrum and discrete harmonic frequencies loading. Rayleigh damping coefficients were evaluated for later use in FE models. Panel health was checked periodically through a power spectral density response on white noise signal. The ultrasonic NDT instruments were used for monitoring of relevant delamination increment. No increments of delamination were detected. The available acoustic power was below the fatigue limit of the tested structure.
\end{abstract}

\section{Introduction}

There are structural elements on aircraft that may be exposed to high intensity sound levels. One of them is an air inlet duct of the jet engine. With its very complex geometry, Figure 1, the air inlet duct is predestined to be made by composite technology.
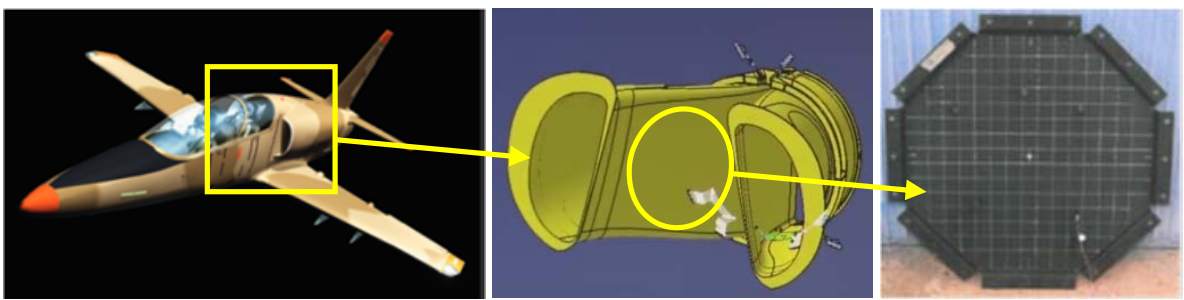

Fig. 1. Relations between the airframe, structural part and test specimen.

High sound levels may cause vibrations of the structural element which are excited by the sound pressure fluctuations. The vibration may lead to cracks in the structural elements

\footnotetext{
${ }^{1}$ Corresponding author: behal@vzlu.cz
} 
and already existing cracks may grow. Regarding to composite structural elements the defects of delamination type can be under consideration above all.

A design of airframe structures has to be accompanied by strength analyses and composite material properties testing, [1], on different complexity levels: on coupons level, [2], structural details level, [3], and finally on the airplane component level, [4].

The paper introduces an approach to the acoustic fatigue test, including selection of its conception, control and measurement systems and acoustic power source. Some experiences with composite panel testing will be noted.

\section{Acoustic fatigue test arrangement}

A simple acoustic fatigue test facility was designed and manufactured to support fatigue analysis of the air duct. The attention was focused on a progressive wave tube (PWT) concept, $[5,6]$, with normal or grazing acoustic wave incidence. Reverberant or anechoic chambers were refused with regard to inevitable construction works in a test lab.

Compared to a reverberant chamber, the PWT allows the excitation of the test specimen at discrete frequencies which can provide data for both the evaluation of material characteristics and the verification of the FE analysis results, whereas loading under random or given spectrum of acoustic intensities is possible as well.

\subsection{Concept selection}

\subsubsection{Normal and grazing acoustic wave incidence}

The concept selection was based on the FE analyses of simple principle models, created in ABAQUS program, [7]. A cube of acoustic medium in contact with a tested panel was excited by acoustic waves on opposite face for the normal acoustic wave incidence, Figure $2 \mathrm{a}$, and on a side face of the cube for the grazing acoustic wave incidence, Figure $2 \mathrm{~b}$.

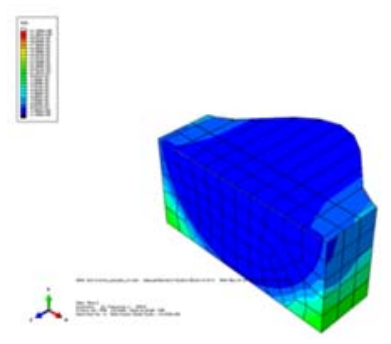

a)

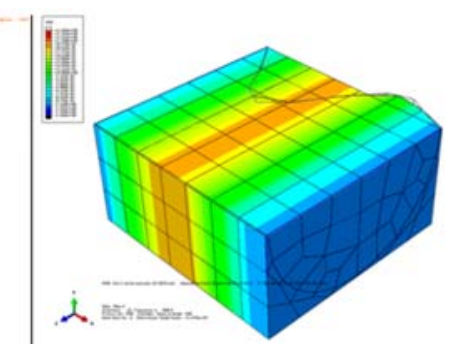

b)

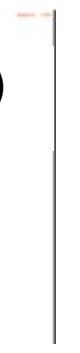

Fig. 2. FE principle models with normal and grazing acoustic wave incidences.

The FE analyses showed that normal wave incidence can excite the symmetrical harmonic forms only. Moreover, there is a formation of standing waves in dependence on the frequency of the acoustic waves and on the distance between the tested panel and the face of the cube, on which is the acoustic pressure applied.

The concept of PWT with grazing acoustic waves was realized.

\subsubsection{PWT acoustic optimization}

The design of the PWT was subordinated to dimensions of the composite panel, which were primarily proposed for the impact tests. The main intent of the PWT design was to get planar grazing waves up to maximal possible frequency. 
The cross section of the tube is rectangular. Its height is determined by the panel dimension including its clamping sections. The width was adjusted on basis of the FE analyses to minimize cross wave reflections resulting in the planar wave disintegration, Figure 3.
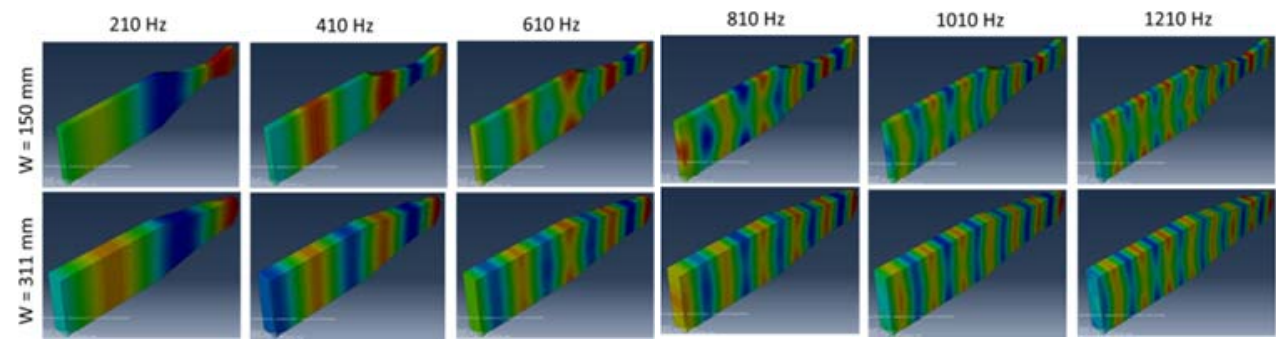

Fig. 3. Cross section width (W) effect on the on the frequency of the planar wave disintegration.

The tube was made from thick medium-density fibreboard (MDF) plates with high coefficient of internal damping to minimize its self-excitation.

Two electro-magnetic speakers with standard sensitivity of $100 \mathrm{~dB}$ were embedded into the PWT to amplify the acoustic wave intensity. Maximal acoustic intensity of $132 \mathrm{~dB}$ was measured when $200 \mathrm{~Hz}$ and $1000 \mathrm{~Hz}$ harmonic frequency loading were applied.

Final design of the selected conception was checked by the FE analyses regarding to the planarity of the acoustic waves and to the limit frequencies of their coherency, image a) in Figure 4, and compared with measured acoustic intensities on the manufactured acoustic fatigue test facility, image b) in Figure 4.

The acoustic fatigue test facility with installed composite panel, the control unit and two high-power amplifiers are introduced in Figure 5.

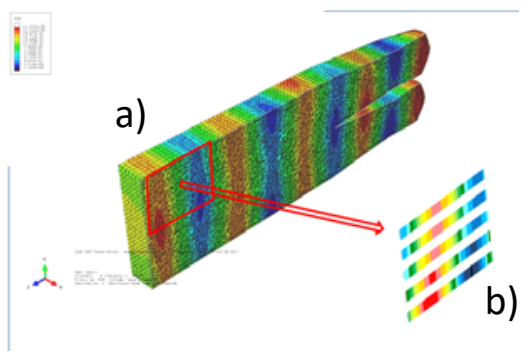

Fig. 4. The FE analysis of the grazing acoustic waves, image a), in comparison to acoustic field intensity measurement, image b).

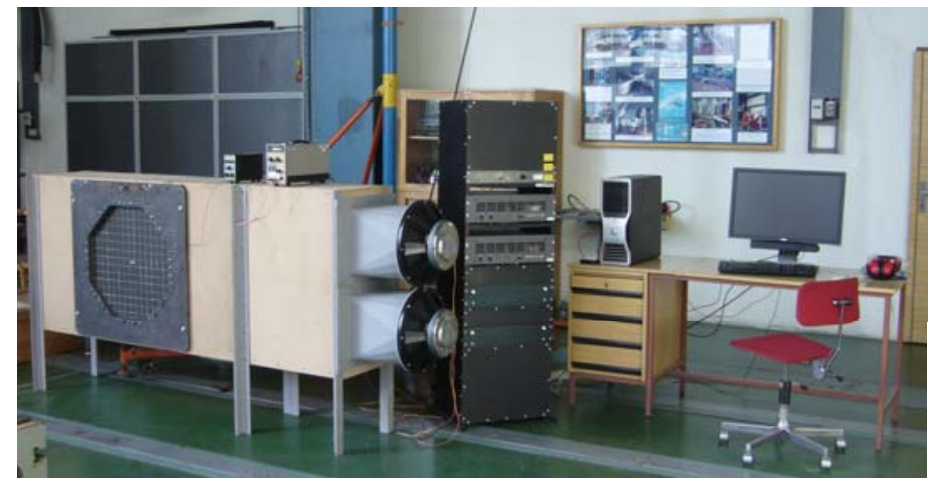

Fig. 5. Acoustic fatigue test facility. 


\subsection{Specimen}

A set of flat composite panels, see Figure 1 above, were designed with different fibre layups. The panels were manufactured in Aero Vodochody Aerospace company using Hexply 8552/AGP193-PW prepreg. Their thickness was in range from 1.8 to $3.5 \mathrm{~mm}$ and their width was $600 \mathrm{~mm}$. Production imperfections of delamination type were simulated by a thin non-adhesive insert film. Behaviour of the composite panel without any significant defects and panel with a production imperfection were tested.

\section{Acoustic loading}

Three types of acoustic spectra were applied: white noise, engine spectrum simulation and selected harmonic frequencies.

All spectra and frequencies were applied with the same range of maximal and minimal levels of control signals, along with constant setting of the high-power amplifier unit. The composite panel behaviour was analysed in frequency domain (upper diagrams in Figures 6 to 8). The loading sequences and acceleration in centre of panel are presented in time domain (lower diagrams in Figures 6 to 8).

\subsection{Modal characteristics measurement}

\subsubsection{White noise}

During acoustic fatigue testing, the panel was periodically checked for its power spectral density response on acoustic loading using a white noise signal, Figure 6.

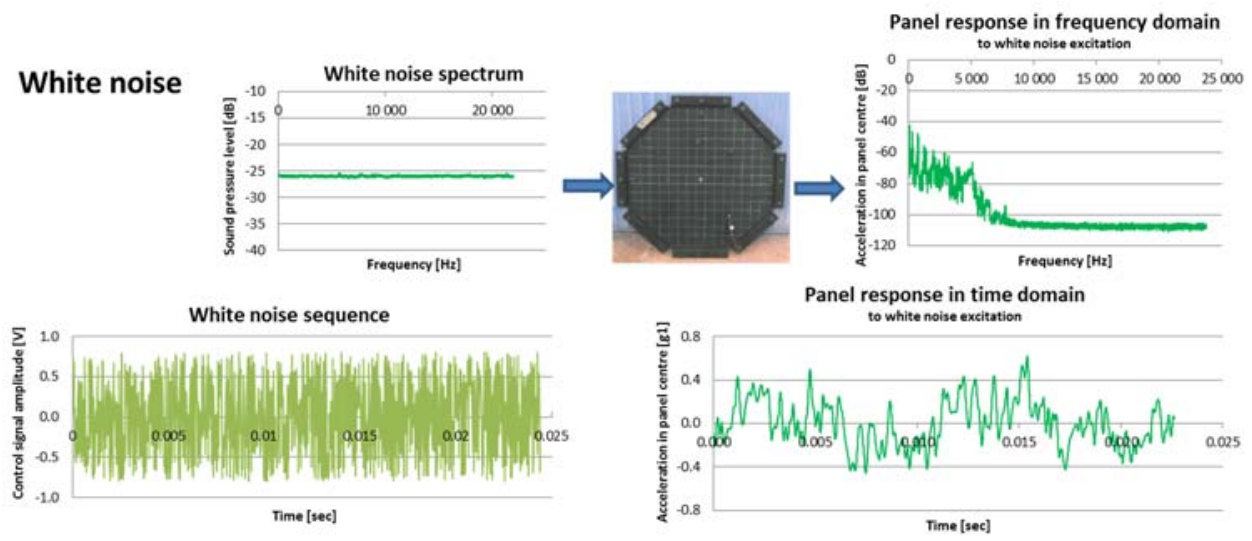

Fig. 6. Dynamic behaviour of the panel under loading by white noise sequence.

The power spectral density (PSD) of the white noise sequence is constant and independent on frequency. White noise loading is advantageous way to evaluate a transient function of the tested system - the shape of the transfer function corresponds directly to PSD which can be evaluated from the response of the tested system. Single peaks on the evaluated PSD correspond to natural frequencies of the tested system.

Regarding to the tested panel, it is necessary to account for the upper frequency limit of speakers, which is about $5 \mathrm{kHz}$. This induces a downward of the panel response between 5 to $8 \mathrm{kHz}$ frequencies in Figure 6. 


\subsubsection{Engine spectrum simulation}

The ability of the PWT to simulate real acoustic conditions in the air duct was tested under loading by a measured sequence of the engine noise, Figure 7.

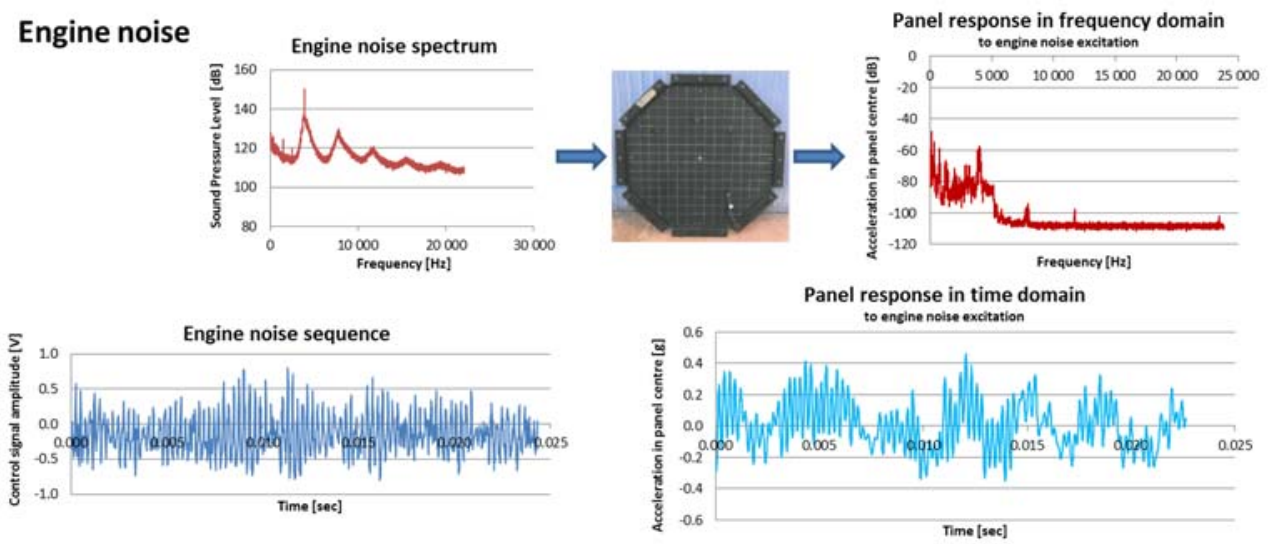

Fig. 7. Dynamic behaviour of the panel under loading by engine noise sequence.

The peak of acoustic noise about $4 \mathrm{kHz}$ corresponds to a turbofan blade-passage frequency (a product of the rotational speed of the fan and the number of fan blades) and is well transferred onto the panel. The $2^{\text {nd }}$ and $3^{\text {rd }}$ harmonic of the blade-passage frequency are noticeable as well, but with decreased levels due to transfer function of the applied speakers, as mentioned in 2.3.1.

\subsubsection{Discrete harmonic frequency}

There are two reasons to apply loading sequence of a discrete harmonic frequency:

- The natural frequency can be used to excite the panel deformation in resonance, which is effective way to get maximal panel deflections with minimal acoustic intensity, especially for the fatigue test.

- Parameters of the FE model of the tested panel can be easily adjusted to credibly simulate the panel behaviour under acoustic loading comparing to measured data.
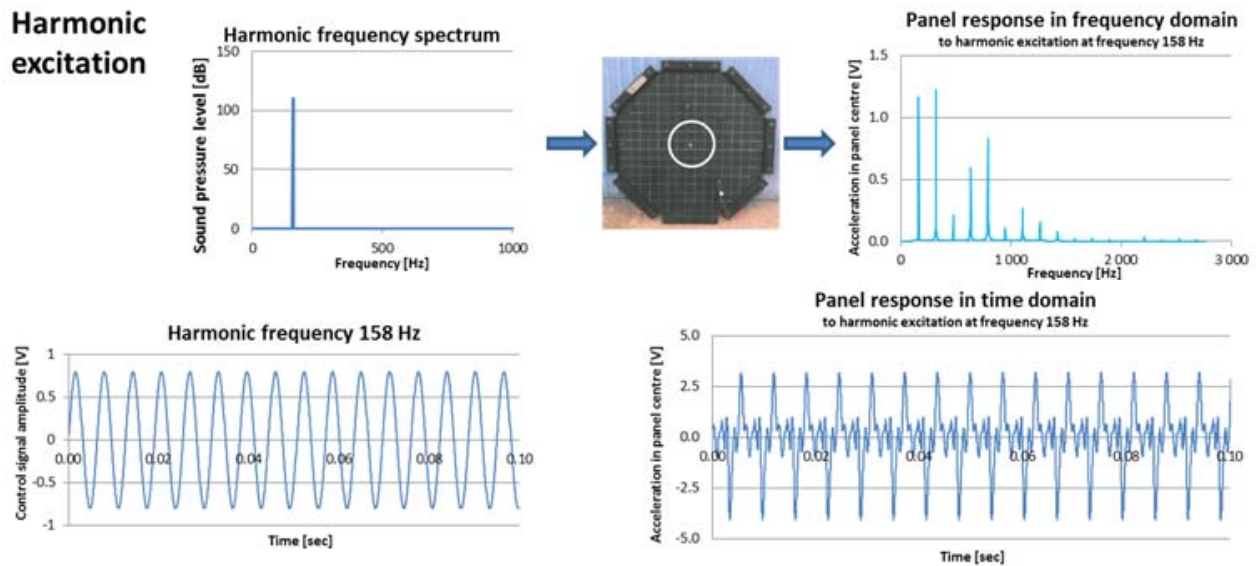

Fig. 8. Dynamic behaviour of the panel under $158 \mathrm{~Hz}$ harmonic loading. 
A complex dynamic behaviour of the composite panel with a central delaminated area is presented in Figure 8 as an example. A lot of higher harmonic modes occur in measured spectrum of acceleration in the panel centre probably due to the interactions between the delaminated surfaces.

\subsection{Identification of damping properties}

There is significant reason for adding damping into the FE model: to limit the amplitude of oscillations resulting from the FE analysis to a real range which is relevant to the physical nature of the task. RAYLEIGH damping model, Figure 9, was chosen and applied according to the requirements of the ABAQUS code, [7].

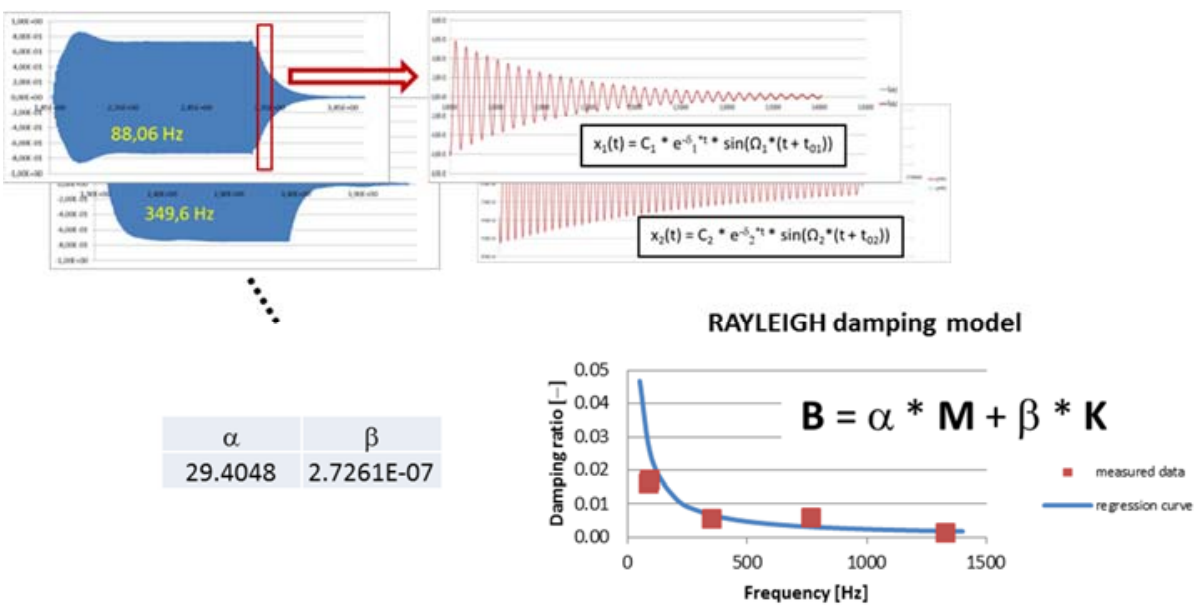

Fig. 9. RAYLEIGH damping model evaluation for tested composite panel.

\subsection{Effect of delamination on the panel dynamic behaviour}

Deflection curve of the tested panel was measured under cyclic loading at discrete frequencies. Two significant modes are presented in Figure 10.

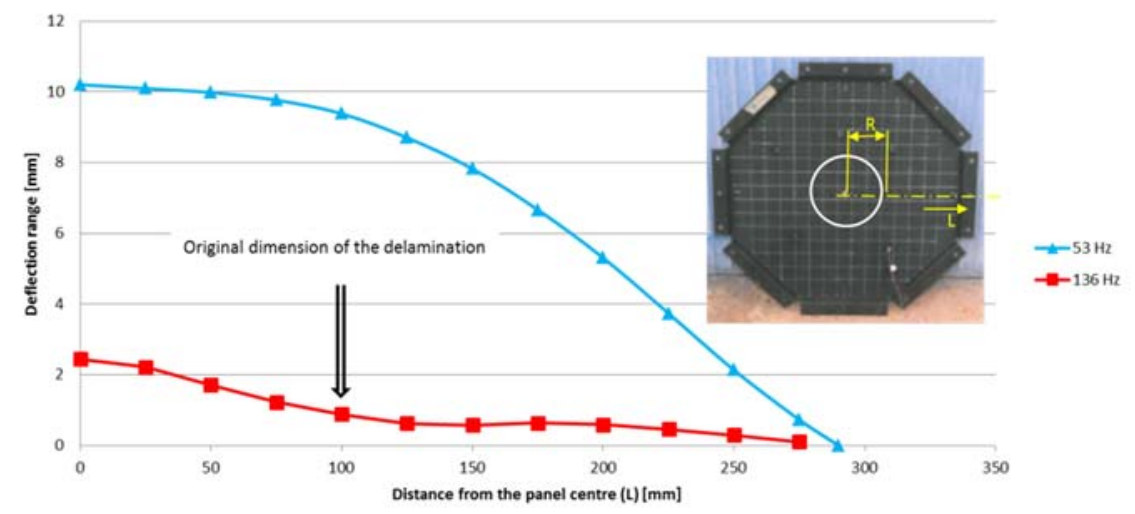

Fig. 10. Two significant deflection modes of composite panel with central delamination at $53 \mathrm{~Hz}$ and $136 \mathrm{~Hz}$ frequencies. 
Two dynamic systems can be identified: panel oscillations as a whole, and independent oscillations of the separated parts of the panel whereas the intact part of the panel is practically inactive.

Regarding to the acoustic fatigue test with the aim to get information about the delamination growth rate, the second case of loading is more interesting: the curvature of the deformed panel at the delamination border is higher than in the first case, thus the bending moment in this point is higher as well. But there was not any reserve of the PWT acoustic power to get the higher deflection of the panel and propagation of the delamination because the frequency corresponding to this oscillation mode is higher.

\subsection{NDT indications}

During the test the ultrasonic NDT instruments were periodically used to evaluate relevant delamination increments. No increments of the original delaminated area were detected.

\section{FE simulations}

The FE analyses will be necessary for fatigue life evaluation support because of the small acoustic power of the PWT. First proposal of the FE model was compared with real product behaviour, Figure 11.

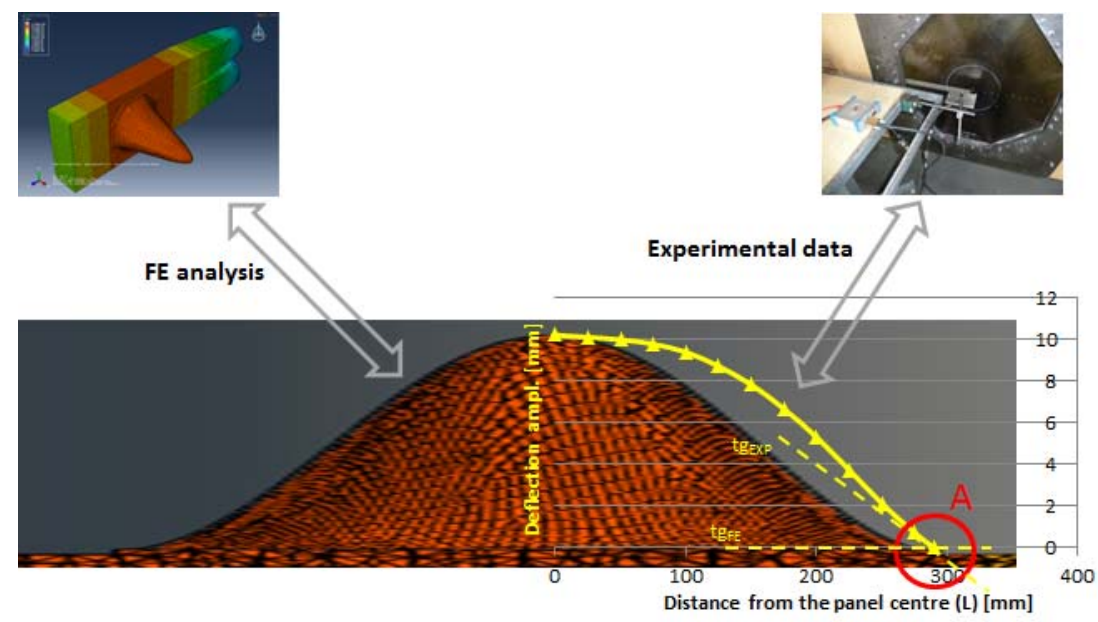

Fig. 11. Comparison of the composite panel deflection curves evaluated by the FE analysis with the PWT measurement.

There is difference between the measured deflection curve and the panel deformation evaluated by the FE analysis. The attention should be focused on the A point, the end of clamping area. It is obvious the tangent lines to the both curves are significantly different. It can be stated that:

- The FE model boundary conditions (all translational and rotational degrees of freedom set to zero) on clamped panel boundary are not convenient, or

- The panel works like a membrane rather than a plate because of their small bending stiffness.

These hypotheses will be tested in next work. 


\section{Conclusions}

The acoustic fatigue test equipment based on progressive wave tube concept was developed, manufactured and tested. Acquired experience allows to state that the used acoustic sources:

- Electro-magnetic speakers do not supply sufficient intensity of acoustic noise to simulate real level of jet engine noise even if all acoustic power is concentrated into a wave tube of limited cross section area.

- The frequency spectrum, which can be excited on the test specimen, is limited according to speaker frequency range.

On the other side, dynamic characteristics of tested specimen can be evaluated to support the FE analyses which appear to be a base for the proof of fatigue life of composite air duct:

- The specimen transfer function can be verified by white noise testing.

- The discrete harmonic frequency can be used to find resonance conditions and provide data for the FE model verification in regard to the stiffness and mass simulation.

- Structural and material damping properties can be evaluated for the FE model to give real values of deformations and stresses of the part.

From the fatigue point of view can be stated, that applied acoustic loading, regarding to the tested panel with the simulated production imperfection, was below the fatigue limit of the structure.

\section{References}

1. AMC 20-29 - Composite Aircraft Structure, EASA, Annex II to ED Decision 2010/003/R of 19/07/2010 (2010)

2. M. Kadlec, J. Šedek, Experimental stress analysis Conf. Proc., Delamination Growth under Mixed Mode I - Mode II Fatigue Loading in Polymer Composite, 56, 168 (2018)

3. R. Doubrava, J. Raška, M. Oberthor, Bird strike test and analysis of flat composite panels, (Poster on 8th International Conference on Fracture of Polymers, Composites and Adhesives, Les Diablerets, Switzerland, 2017)

4. R. Doubrava, M. Oberthor, P. Bělský, M. Dvořák, K. Doubrava, Experimental stress analysis Conf. Proc., Experimental verification of jet engine composite inlet for bird and hail stone impact resistance, 56, 63 (2018)

5. D.M. Forney, Acoustical fatigue test procedures used in aircraft industry and their limitations, (Material Laboratory Wright Air Development Center, Report No. WADC TR 59-676, p. 339-378, 1962)

6. R.A. Bianchi, R.T. Bradshaw, J.H. Farrell, F.E. Reed: Survey and evaluation of sonic fatigue testing facilities, (Wright-Patterson Air Force Base, Technical Report No. ASD-TR-61-185, 1962)

7. Abaqus/CAE User's Guide, version 6.13.3, Dassault Systèmes (2013) 